\title{
Safety and efficacy of venom immunotherapy: a real life study
}

\author{
Agnieszka Kołaczek, Dawid Skorupa, Monika Antczak-Marczak, Piotr Kuna, Maciej Kupczyk \\ Clinical Department of Internal Medicine, Asthma and Allergy, Medical University of Lodz, Lodz, Poland \\ Adv Dermatol Allergol 2017; XXXIV (2): 159-167 \\ DOI: https://doi.org/10.5114/ada.2017.67082
}

\begin{abstract}
Introduction: Venom immunotherapy (VIT) is recommended as the first-line treatment for patients allergic to Hymenoptera venom.

Aim: To analyze the safety and efficacy of VIT in a real life setting.

Material and methods: One hundred and eighty patients undergoing VIT were studied to evaluate the safety, efficacy, incidence and nature of symptoms after field stings and adverse reactions to VIT.

Results: Significantly more patients were allergic to wasp than bee venom (146 vs. $34, p<0.0001)$. Early and late side effects were more common during the maintenance (48 patients, $26.7 \%$ ) than during the induction of VIT (32 patients, 17.8\%), were more frequent in patients allergic to bees, and were not associated with angiotensin convertase inhibitors (ACEi) or $\beta$-adrenergic antagonists use. Systemic reactions were observed in 4 individuals on wasp VIT (2.7\%) and in 6 patients allergic to bees (17.65\%). The VIT was efficacious as most patients reported no reactions $(50 \%)$ or reported only mild local reactions $(43.75 \%)$ to field stings. The decrease in slgE at completion of VIT correlated with the dose of vaccine received $(r=0.53, p=0.004)$. Beekeeping $(\mathrm{RR}=29.54, p<0.0001)$ and female sex $(R R=1.27, p=0.033)$ were associated with a higher risk of venom allergy.

Conclusions: Venom immunotherapy is highly efficacious and safe as most of the adverse events during the induction and maintenance phase are mild and local. Side effects of VIT are more common in subjects on bee VIT. Beekeeping and female sex are associated with a higher risk of allergy to Hymenoptera venom.
\end{abstract}

Key words: venom immunotherapy, side effects, bee, wasp, Hymenoptera.

\section{Introduction}

Stings by Hymenoptera insects are relatively common in the population and may lead to a range of reactions from mild and local symptoms to life-threatening anaphylaxis. In Europe mainly honeybee (Apis mellifera) and wasp (US nomenclature: yellow jacket, Vespula germanica and vulgaris) are responsible for those incidents [1]. The allergy to Hymenoptera venom affects around $15-30 \%$ of the general population (as confirmed by skin prick tests or slgE) and the frequency of systemic reactions due to Hymenoptera venom allergy varies between 0.35 to $4 \%$ [2, 3]. The incidents of fatalities due to stings are estimated at 0.03 to 0.48 deaths per 1000000 citizens per year [4]. Venom immunotherapy (VIT) represents an effective causative treatment for these patients preventing further sting-induced anaphylactic reactions. Immunotherapy may be associated with a risk of local and systemic side effects. Adverse reactions during venom immunotherapy are relatively common and are reported in up to $50 \%$ of patients, mostly during the build-up phase. Systemic reactions occur in $12 \%$ to $30 \%[5,6]$ of subjects undergoing VIT, which results in a common belief that VIT is potentially dangerous. As a consequence, a limited access to this life-saving procedure may be seen. What is striking, a high level of variations (0-46\%) and inconsistency in the side-effects frequency is reported in the literature [7-10]. Moreover, there are just a few comprehensive studies dedicated to this topic [11-16].

\section{Aim}

Thus, the goal of this study was to analyze safety, efficacy and adherence to treatment in a real-life setting in a group of patients who underwent immunotherapy due to bee or wasp venom allergy. The incidence and nature of symptoms after field stings and adverse reactions to

Address for correspondence: Maciej Kupczyk MD, PhD, Clinical Department of Internal Medicine, Asthma and Allergy, Medical University of Lodz, 22 Kopcinskiego St, 91-350 Lodz, Poland, phone: +48 4267769 50, e-mail: maciej.kupczyk@umed.lodz.pl Received: 10.06.2015, accepted: 10.03.2016. 
VIT were studied. Factors associated with a higher risk of allergy to Hymenoptera venoms were also analyzed.

\section{Material and methods}

One hundred and eighty adult patients undergoing VIT at the Department of Internal Medicine, Asthma and Allergy, Medical University of Lodz, Poland, including 146 subjects allergic to the wasp venom and 34 subjects allergic to bee venom, were included into the study. Their medical histories were analyzed retrospectively. Due to the design of the study, no institutional review board approval was required. All patients included in the study were undergoing VIT against Hymenoptera venom (honey bee or wasp venom) due to systemic reactions after stings. The following criteria in the baseline patients' characteristics were taken into consideration: sex, age, the type of the insect, levels of slgE, Muller's scale after stings before VIT, the criterion of VIT termination. Patients were also asked about the association with beekeeping (questions of the beekeeper in the family and in the close neighborhood) and medications taken - angiotensin convertase (ACE) inhibitors and $\beta$-adrenergic antagonists.

\section{Venom immunotherapy}

Depending on the type of allergy, specific and standardized allergen extracts of bee (Apis mellifera) or wasp (Vespula spp.) venom were used (Pharmalgen ${ }^{\oplus}$, Alutard ${ }^{\oplus}$, or Venomenhal ${ }^{\oplus}$ ). In majority of subjects but one, an ultra-rash protocol was used at the induction phase of VIT. The vaccine was administered subcutaneously with the increasing doses of: 1, 10, 20, 30 and finally $50 \mu \mathrm{g}$ in the 30 min' intervals of time (111 $\mu \mathrm{g}$ in total). After induction patients were receiving a maintenance dose $(100 \mu \mathrm{g})$ of a vaccine every 4-6 weeks, depending on the tolerance and duration of treatment. All patients undergoing venom immunotherapy at our center received oral antihistamines as a pretreatment before the ultra-rush induction phase and during the maintenance treatment.

\section{Data collected during the course of venom immunotherapy}

The dose of the vaccine after each injection, all adverse reactions, wasp or bee-stings during VIT, and symptoms after stings were reported in the medical histories and classified according to the four-point Muller's scale. Side effects during induction of VIT and during maintenance treatment of VIT were classified as early (occurring up to 30 min after the injection) and late (appearing after $30 \mathrm{~min}$ ). The early side effects have been registered in the hospital. Patients were obliged to report all the late side effects, potential field stings during VIT and symptoms after stings on the next visit in the clinic. Specific
IgE levels at the baseline, during and after VIT were analyzed.

\section{Statistical analysis}

To assess the relationship between two independent category groups of data, the $\chi^{2}$ test or Fisher exact test were used. For the independent variables, including average VIT time and injections number, summary dose of vaccines, Muller's median scale and average slgE titer, the Mann-Whitney and $t$-test for independent means were used. To compare the decrease in slgE after completed VIT, Wilcoxon test was used. Analysis of the correlation was made, depending on the type of data, with the Pearson's (for linear correlation) and Spearman's correlation (other kinds of correlation). Statistical analysis was performed using GraphPad Prism version 5. The confidence interval was set at $0.95(95 \% \mathrm{Cl})$ and the level of significance was set at $p<0.05$.

\section{Results}

One hundred and eighty patients, aged $15-77$ years, were included in the study. Demographic data and baseline characteristics of the study group are presented in Table 1. Comparison between subjects allergic to bee and wasp venom is presented in Table 2. There were significantly more patients undergoing VIT due to allergy to wasp than bee venom (146 vs. $34, p<0.0001$ ). The majority of patients included in both groups were women (wasps/bees; 61.6\%/61.8\% of women). Patients allergic to bee venom were significantly younger (44 vs. 50 $y, p=0.042$ ). Average duration of VIT, at the time of the study was 35 months, the average number of injections was 39 and the total dose of vaccine was $2637.75 \mu \mathrm{g}$. Five patients allergic to bee venom were beekeepers and twelve had a relationship with beekeeping. The percentage of patients taking $\beta$-adrenergic antagonists or inhibitors of ACE was over $20 \%$ and was similar in both study cohorts. The adherence to treatment was high as all patients qualified for venom immunotherapy continued the treatment in line with the timelines suggested. Two subjects were referred to other allergy centers due to a change of the residence.

Symptoms after stings for all patients before and during VIT are presented in Figure $1 \mathrm{~A}$ and $\mathrm{B}$. All patients qualified for the study presented systemic reactions. Median of Muller's scale before VIT was 3 and during VIT decreased to 0 in those patients, who have experienced field stings during the VIT (Figure 1C, D).

\section{Side effects during venom immunotherapy for all patients}

Side effects during VIT were reported by 64 patients (43.8\% of all patients). Early and late side effects were more common during the maintenance phase of VIT (48 pa- 
Table 1. Demographic data and baseline characteristics of the study group

\begin{tabular}{|c|c|c|}
\hline Baseline characteristics of the study group & Number of patients & $\%$ of group \\
\hline Number of patients/female/male & $180 / 111 / 69$ & $100 / 62 / 38$ \\
\hline Age [years] (min.-max.) & $49(15-77)$ & - \\
\hline Number of patients allergic to wasp venom/bee venom & $146 / 34$ & $81 / 19$ \\
\hline Number of beekeepers & 6 & 3.33 \\
\hline Number of persons whose husband/wife/neighbors have a bee yard & 12 & 6.67 \\
\hline Median of Muller's scale before VIT & 3 & - \\
\hline Average time of VIT [months] ${ }^{*}$ & 35 & - \\
\hline Average injections number/average total dose of vaccine $[\mu \mathrm{g}]$ & $39 / 2637.75$ & - \\
\hline Scheme of the initial phase of VIT - ultra-rush & 179 & 99.44 \\
\hline Scheme of the initial phase of VIT - cluster other than ultra-rush & 1 & 0.66 \\
\hline Number of patients on ACE inhibitors or $\beta$-adrenergic antagonists during VIT & 37 & 20.56 \\
\hline
\end{tabular}

*Time of observation of the patient during VIT.

Table 2. Baseline characteristics of patients allergic to wasp and bee venom undergoing VIT and subgroup of patients who completed a 5-year cycle of VIT

\begin{tabular}{|c|c|c|c|}
\hline Variable & Wasp & Bee & $P$-value \\
\hline \multicolumn{4}{|l|}{ Patients allergic to wasp and bee venom: } \\
\hline Number of patients & 146 & 34 & $<0.0001^{1}$ \\
\hline Age [years] (min.-max.) & $50(18-77)$ & $44(15-71)$ & $0.042^{2}$ \\
\hline Females & $90(61.6 \%)$ & $21(61.8 \%)$ & $1.0^{2}$ \\
\hline Average time of VIT [months] & 34 & 40 & $0.449^{2}$ \\
\hline Average injections number/average total dose of vaccine $[\mu \mathrm{g}]$ & $37 / 2576.38$ & $47 / 2901.31$ & $0.152 / 0.375^{2}$ \\
\hline Number of beekeepers & $1(0.68 \%)$ & $5(14.71 \%)$ & $0.0012^{1}$ \\
\hline Number of persons related to beekeeping & 0 & $12(35.29 \%)$ & $<0.0001^{1}$ \\
\hline Number of patients taking $\beta$-adrenergic antagonists or inhibitors of ACE & $30(20.55 \%)$ & $7(20.6 \%)$ & $0.66^{1}$ \\
\hline Number of patients stung during VIT & $38(26 \%)$ & $10(29 \%)$ & $0.672^{1}$ \\
\hline Median of Muller's scale after stings before VIT/during VIT & $3 / 0$ & $3 / 0$ & $0.1712 / 0.933^{2}$ \\
\hline Median of IgE before VIT (class)/average IgE before VIT [UI/ml] & $2.55 / 6.19$ & $2.5 / 5.36$ & $0.887^{2}$ \\
\hline \multicolumn{4}{|l|}{ Patients who completed a 5-year cycle of treatment: } \\
\hline Number of patients who completed a 5-year cycle of treatment & 18 & 3 & $0.769^{1}$ \\
\hline Average length of VIT in patients who completed VIT [months] & 69.4 & 94 & $0.039^{2}$ \\
\hline Average injections number in persons who completed VIT & 60 & 96 & $0.056^{2}$ \\
\hline Average total dose of the vaccine in persons who completed VIT [ $\mu \mathrm{g}]$ & 4569.4 & 6046.4 & $0.029^{1}$ \\
\hline $\begin{array}{l}\text { Median IgE after 5-year treatment of VIT (class)/average IgE after 5-year } \\
\text { treatment of VIT [UI/ml] }\end{array}$ & $2.05 / 1.36$ & $2.6 / 2.53$ & $0.064^{2}$ \\
\hline
\end{tabular}


A

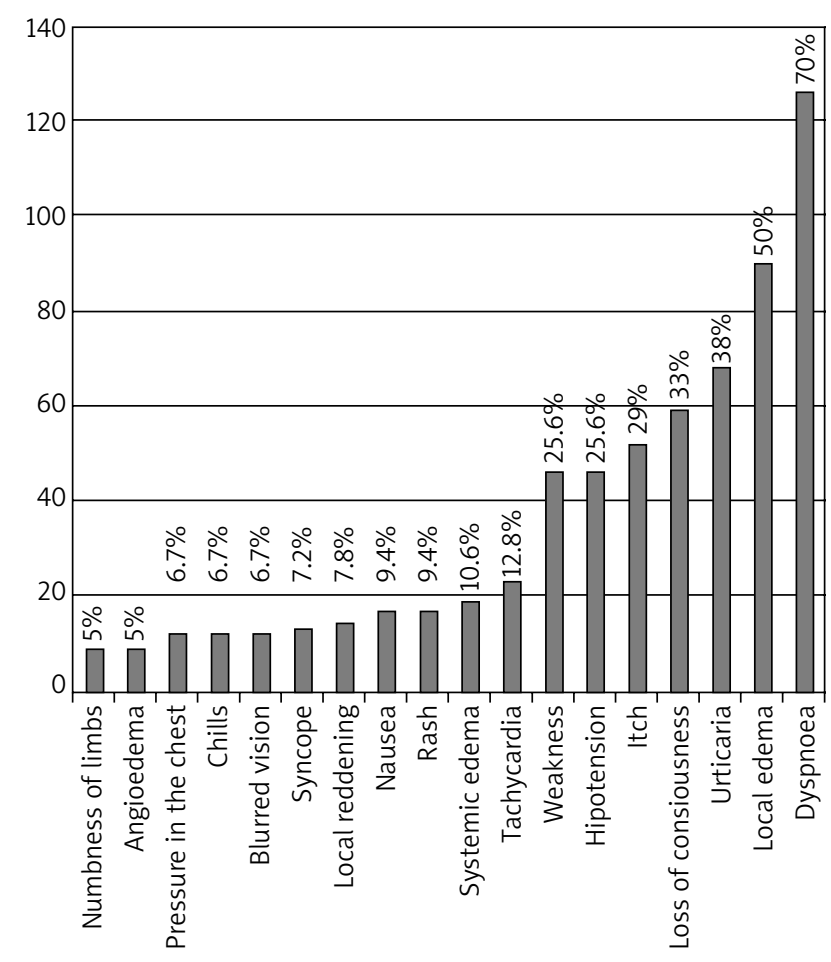

C

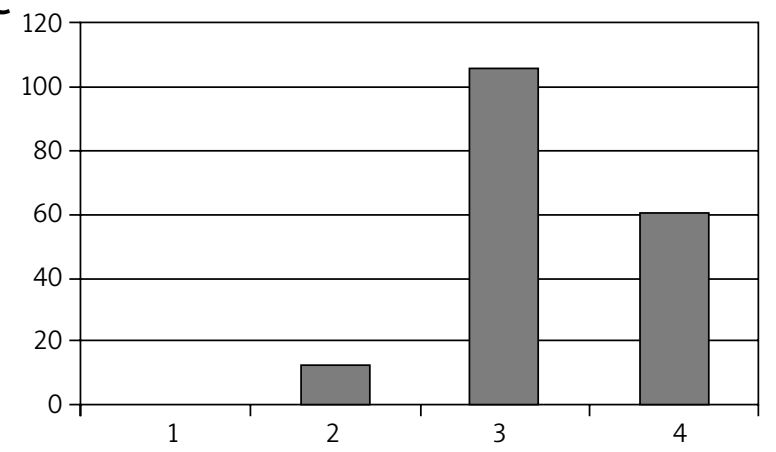

B

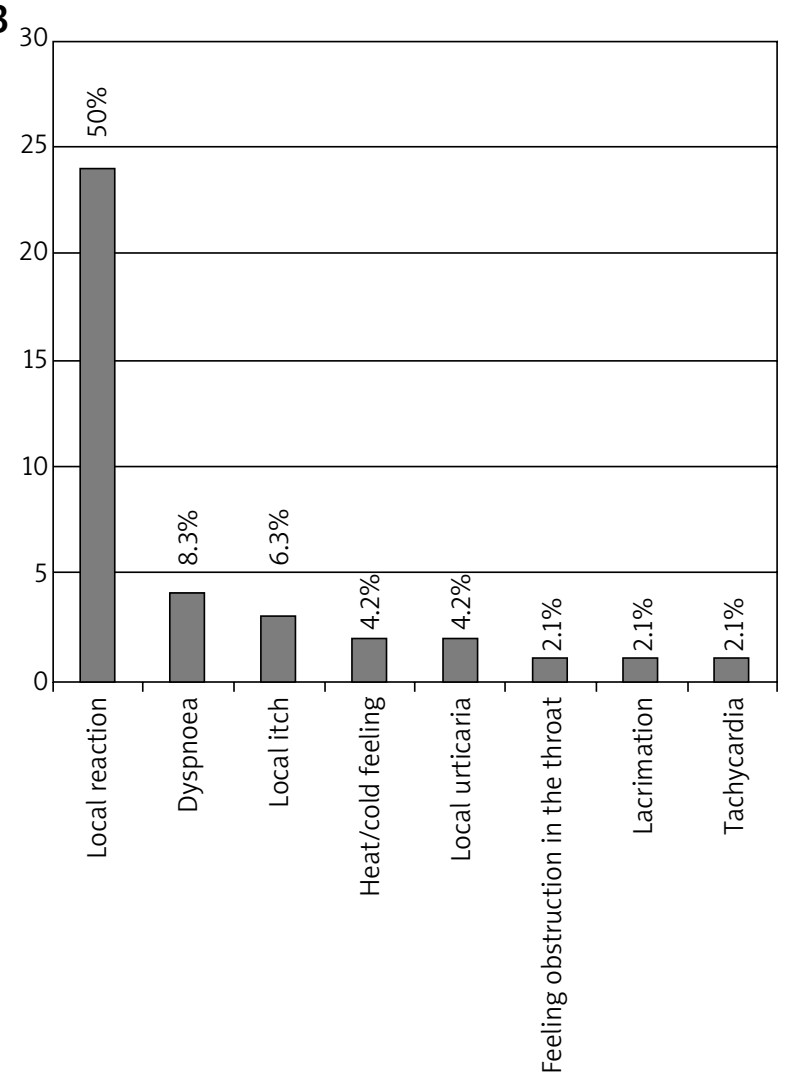

$D_{50}$

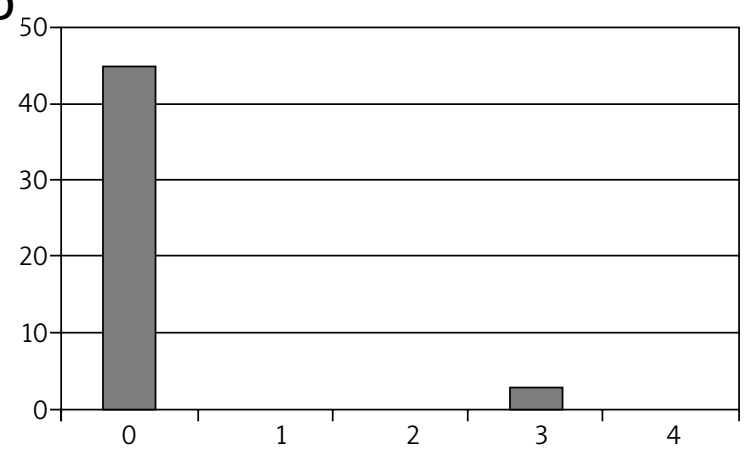

Figure 1. Symptoms after stings for all patients before VIT (A), and symptoms after field stings during VIT (B). Severity of reactions described with Muller's scale for all patients after stings before VIT (C), and after field stings during VIT (D)

tients, $26.7 \%$ of the study group) than during the induction phase (32 patients, $17.8 \%$ ). In detail, during the induction of treatment early side effects were reported by $9.4 \%$ of patients and late side effects were reported by $8.3 \%$ of patients. During the maintenance phase, early side effects were rare (7.2\%), however the late side effects were more frequent (19.4\%). Systemic reactions as side effects during the induction and maintenance phase of VIT were observed in 4 individuals undergoing VIT against the wasp (2.7\%) and in 6 patients allergic to bee venom (17.65\%). Only in 1 case, the administration of adrenaline was needed. Majority of systemic reactions were reported in the induction phase $(1.9 \%$ of injections), not in the maintenance phase $(0.19 \%$ of injections). Most of the systemic reactions (68.2\%) developed within 30 min after the injection. All late systemic reactions (31.8\% of events) were restricted to skin changes (urticaria, flush, reddening of the skin). The frequency and nature of adverse reactions to VIT are presented in Figure 2. Treatment with $\beta$-adrenergic antagonists or ACE inhibitors was not associated with any kind of adverse events during VIT $(p=0.773)$, systemic adverse events during VIT $(p=0.74)$ or more severe symptoms during field stings $(p=0.804)$. 


\section{Comparison of side effects during venom immunotherapy in patients allergic to bee and wasp venom}

Early and late side effects during induction of VIT were more frequent in patients allergic to bees than in patients allergic to wasps. Similarly, during maintenance treatment early and late side effects were more common in patients allergic to bees than in patients allergic to wasps (Table 3). Most of the side effects were local and included local swelling, reddening of the skin and itch at the site of injection (Figure 2). Local urticaria was more frequent as an early side effect during induction of VIT and was more common in patients allergic to bee venom $(p=0.003)$. There were no significant differences in late side effects during induction of VIT in patients allergic to bee and wasp venom. Acute local reaction, weakness, dyspnea, local itch were more frequent as early side effects in patients allergic to bee venom during the maintenance treatment $(p<0.05)$. Dyspnea, rhinitis and abdominal pain were more common as late side effects in patients allergic to bee venom during the maintenance phase ( $p=0.003$ ). Analyzing stings before VIT, blurred vision $(p=0.02)$ and hypotension $(p=0.02)$ were more frequent in patients allergic to wasp venom.

\section{Side effects during venom immunotherapy depending on sex}

In patients allergic to wasp venom, early and late side effects during the build-up and maintenance phase of VIT were more frequent in women. In contrast, in subjects allergic to bees both early and late side effects dur-

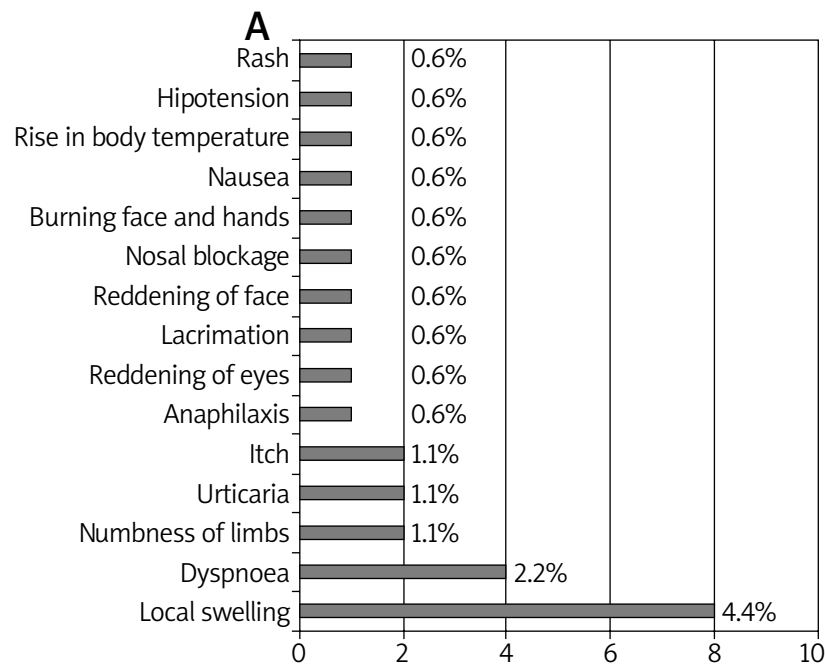

\section{C}

Burning face $0.6 \%$

Wheeze $0.6 \%$

Dysphonia $0.6 \%$

Diarrhea $0.6 \%$

Vomiting $0.6 \%$

Sensation of obstruction in the throat $0.6 \%$

Cough $0.6 \%$

Abdominal pain $0.6 \%$

Nosal blockage $0.6 \%$

Syncope $=0.6 \%$

Hipotension $0.6 \%$

Dizziness $0.6 \%$

Scotoma $0.6 \%$

Heat feeling $0.6 \%$

Reddening of the skin \begin{tabular}{ll}
\hline & \\
\hline
\end{tabular}

Itch of limbs and face \begin{tabular}{ll}
\hline & $1.7 \%$ \\
\hline
\end{tabular}

Dyspnoea $\quad 1.7 \%$

Weakness $1.7 \%$

Local swellin

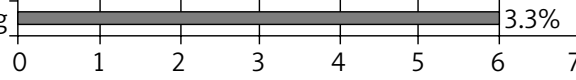

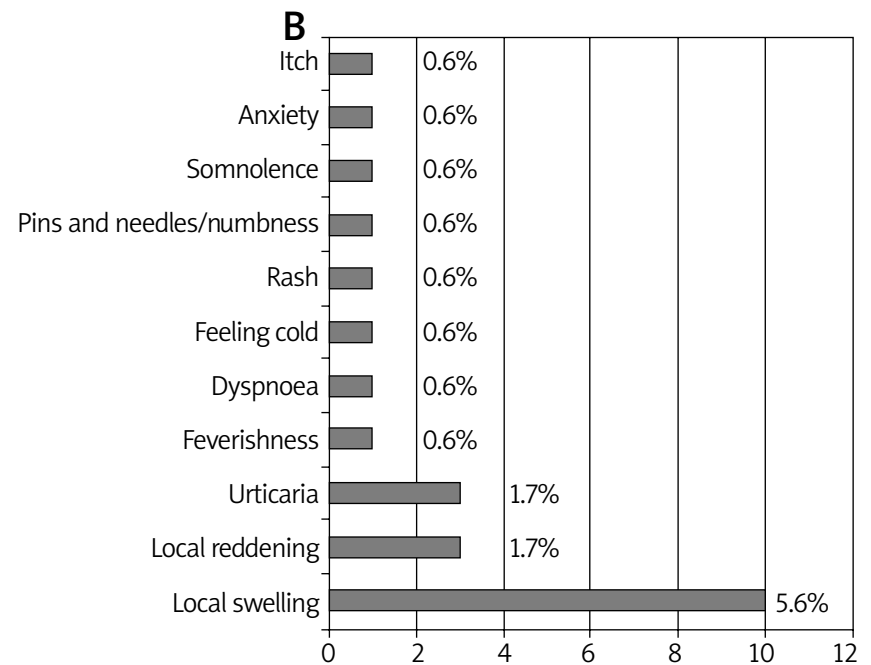

D

Attack of atrial fibrillation $0.56 \%$

Diarrhea $0.56 \%$

Blurred vision $30.56 \%$

Hyperhidrosis $\sqsupset 0.56 \%$

Chills $\sqsupset 0.56 \%$

Fever $-0.56 \%$

Rhinitis $\square 1.1 \%$

Tightness in the throat $\square 1.1 \%$

Weakness $\square 1.1 \%$

Anxiety $\square 1.1 \%$

Numbness of limbs $=1.1 \%$

Dyspnoea $=1.1 \%$

Abdominal pain $\square 1.1 \%$

Headache $\quad 1.7 \%$

Urticaria $=1.7 \%$

Drug eruption $=2.2 \%$

Itch $2.8 \%$

Reddening of the skin $3.3 \%$

Local swelling

0

Figure 2. Side effects during VIT: early side effects during induction of VIT (A), late side effects during induction of VIT (B), early side effects during maintenance treatment of VIT (C), and late side effects during maintenance treatment of VIT (D) 
Table 3. Side effects (SE) during VIT for patients allergic to bee and wasp venom, side effects during VIT depended on sex (wasps), side effects during VIT depended on sex (bees)

\begin{tabular}{|c|c|c|c|c|}
\hline \multirow{2}{*}{$\begin{array}{l}\text { Side effects (SE) during VIT for the whole study } \\
\text { cohort }\end{array}$} & \multicolumn{2}{|r|}{ Wasps } & \multicolumn{2}{|r|}{ Bees } \\
\hline & $N$ & $\%$ of patients allergic to wasps & $N$ & $\%$ of patients allergic to bees \\
\hline SE during VIT & 50 & 34 & 15 & 44 \\
\hline Early SE during induction of VIT & 12 & 8.22 & 5 & 14.71 \\
\hline Late SE during induction of VIT & 11 & 7.53 & 4 & 11.76 \\
\hline Early SE during maintenance treatment of VIT & 5 & 3.42 & 7 & 20.59 \\
\hline Late SE during maintenance treatment of VIT & 27 & 18.48 & 8 & 23.53 \\
\hline \multirow{3}{*}{$\begin{array}{l}\text { Side effects (SE) during VIT in patients allergic } \\
\text { to wasp venom }\end{array}$} & \multicolumn{4}{|c|}{ Wasps } \\
\hline & \multicolumn{2}{|r|}{ Women } & \multicolumn{2}{|r|}{ Men } \\
\hline & $N$ & $\%$ of women & $N$ & $\%$ of men \\
\hline \multicolumn{5}{|l|}{ SE during VIT } \\
\hline Early SE during induction of VIT & 9 & 8.11 & 3 & 5.36 \\
\hline Late SE during induction of VIT & 11 & 9.91 & 0 & 0 \\
\hline Early SE during maintenance treatment of VIT & 5 & 4.5 & 1 & 1.79 \\
\hline Late SE during maintenance treatment of VIT & 18 & 16.22 & 9 & 16.07 \\
\hline \multirow{3}{*}{$\begin{array}{l}\text { Side effects (SE) during VIT in patients allergic } \\
\text { to bee venom }\end{array}$} & \multicolumn{4}{|c|}{ Bees } \\
\hline & \multicolumn{2}{|r|}{ Women } & \multicolumn{2}{|r|}{ Men } \\
\hline & $N$ & $\%$ of women & $N$ & $\%$ of men \\
\hline Early SE during induction of VIT & 3 & 2.7 & 2 & 15.38 \\
\hline Late SE during induction of VIT & 3 & 2.7 & 1 & 7.7 \\
\hline Early SE during maintenance treatment of VIT & 4 & 3.6 & 3 & 23.08 \\
\hline Late SE during maintenance treatment of VIT & 6 & 5.41 & 2 & 15.4 \\
\hline
\end{tabular}

ing induction and maintenance of VIT were more common in men (Table 3).

\section{Field stings during venom immunotherapy}

Ten patients allergic to bee venom and 38 allergic to wasp venom were stung during VIT. The number of stings during VIT by wasps was 52 and by bees reached 22. Subjects allergic to wasp venom were stung usually in August, while those allergic to bee venom in July (Figure 3). Most of patients reported no reactions (50\% of patients stung during VIT) or reported only mild immediate local reactions (43.75\%). None of patients allergic to bee venom reported anaphylaxis, but three patients allergic to wasp venom (7.9\%) had systemic reactions. All of those subjects with a systemic reaction on VIT received doubled dose $(200 \mu \mathrm{g})$ of vaccine and did not report any further systemic reactions after field stings [1]. A weak reverse correlation between the side effect after stings by bees and wasps classified in the Muller's scale and patient's age was found ( $r=-0.36 ; p=0.007)$, which suggests that younger patients had stronger reactions after field stings during VIT than older subjects.

\section{Sub-group of patients who completed venom immunotherapy}

There were 18 patients allergic to wasp venom and 3 allergic to bee venom who completed at least a 5-year cycle of VIT (Table 2). The average duration of treatment, number of injections and the total dose of vaccine was higher in patients allergic to bee than to wasp venom $(p<0.05)$. A significant decrease in the slgE levels was found in those patients who completed VIT. In detail, average slgE in the wasp VIT group decreased from 6.19 IU/ $\mathrm{ml}$ (before VIT) to $1.36 \mathrm{IU} / \mathrm{ml}$ (after 5-year VIT). Average slgE in the bee VIT group decreased from $5.36 \mathrm{IU} / \mathrm{ml}$ to $2.53 \mathrm{IU} / \mathrm{ml}$. The decrease in slgE titer correlated with the total dose of vaccine received $(r=0.53, p=0.004)$.

\section{Factors associated with a higher risk of Hymenoptera venom allergy}

Beekeeping is associated with a higher risk of bee venom systemic reactions $(R R=29.54, p<0.0001)$. Female sex seems to be associated with a higher risk of any Hymenoptera venom systemic reactions $(\mathrm{RR}=1.27, p=$ 0.033). Female sex seems to be associated with a higher 

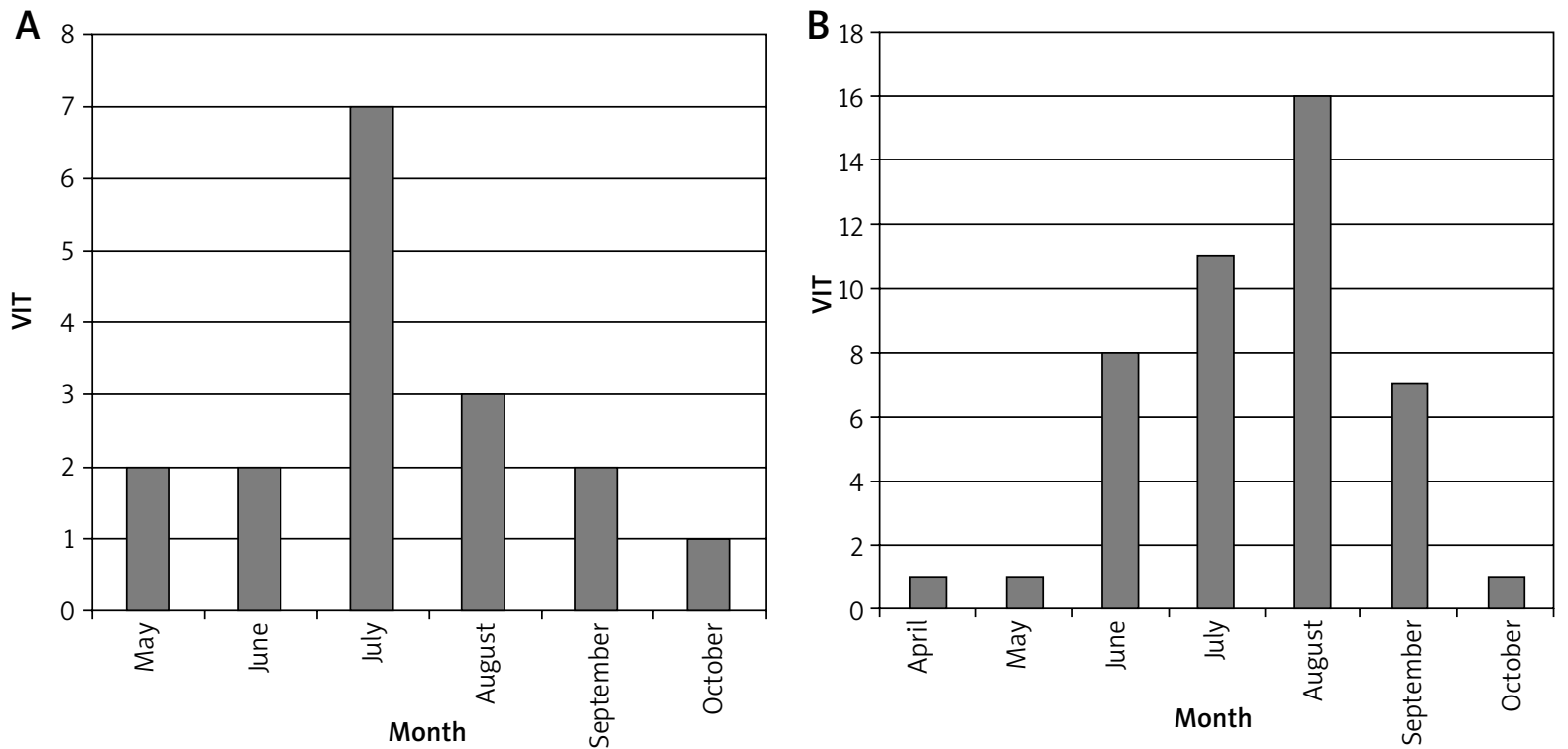

Figure 3. Stinging during VIT for patients allergic to bee venom (A) and wasp venom (B)

risk of development of wasp venom allergy $(R R=1.27$, $p=0.045$ ). A similar trend, however not reaching statistical significance, was found in women allergic to bee venom.

\section{Discussion}

Venom immunotherapy is the only causal treatment for patients allergic to Hymenoptera venom which is a potentially life-threatening condition. Patients allergic to bee or wasp venom, who have experienced a systemic reaction after sting and are not referred to receive VIT, are at a significant (40-75\%) risk of the same or more severe reaction after another sting $[6,17]$. The effectiveness of VIT ranges from 97.3\% [16] to 93.8\% [8] and the risk of systemic reactions after field stings in subjects treated with immunotherapy decreases to $6 \%$. In a multicenter study conducted in 2013, the VIT failure rate (defined as a systemic reaction after a field sting by the culprit insect) reached $6.2 \%$, as 22 out of 357 patients had systemic reactions [8]. In another study, the VIT failure rate was estimated at $6.5 \%$ of all treated cases [9]. This is confirmed by results of our study as in our cohort $3(6.25 \%)$ patients experienced systemic reactions after field stings during VIT. All 3 reactions were graded 3 in Muller's scale and were reported in patients allergic to wasp venom. Patients with a systemic reaction to field sting on VIT received doubled dose $(200 \mu \mathrm{g})$ of vaccine, as suggested by the literature [1] and did not report any further systemic reactions after field stings. In the most comprehensive Cochrane Database systematic review of venom immunotherapy trials published till 2012, 7 studies enrolling 392 patients were analyzed [16]. It has been confirmed that VIT is effective in preventing allergic reactions as only $2.7 \%$ of participants treated had a subse- quent systemic reaction to a sting, compared with $39.8 \%$ of untreated participants. Venom immunotherapy has been found to be effective in preventing large local reactions and improving quality of life of allergic patients.

Unfortunately, VIT may be associated with a risk of adverse events [7]. We have found that side effects during VIT are quite frequent (reported by $43.8 \%$ of patients in our cohort). The range of side effects reported in the literature varies from $17.9 \%$ to $45 \%$ of VIT applications [10-15]. Patients allergic to bee venom were found to be at a higher risk of a systemic allergic reaction during VIT (17.65\% of events vs. $2.7 \%$ in wasp allergic patients). This is in line with several previous reports published to date showing an increased risk of side effects of VIT in bee allergic subjects [10, 13, 18-20]. In the Cochrane Database meta-analysis mentioned above, systemic adverse reactions occurred in $14.2 \%$ of participants treated with bee venom and only $2.8 \%$ in those treated with wasp venom [16]. The described phenomenon may be explained by higher allergenic potency of bee venom itself or the properties of the bee venom extract used for immunotherapy, as it has been proposed previously [18-20].

Almost all but one patient in our cohort were treated with the ultra-rush protocol. Several studies to date proved that cluster protocols, including the rush or ultrarush scheme are significantly safer and much better tolerated by patients in contrast to a traditional protocol [12, 14, 15, 19, 20]. Early and late side effects are more common during the maintenance than during the induction of VIT with the ultra-rush protocol, whereas systemic side effects are significantly more frequent at the induction of VIT. Most of the side effects reported in our study were mild and localized, and included local swelling, reddening of the skin and itch at the site of the injection. Systemic 
reactions as side effects during the induction and maintenance phase of VIT were observed in 4 individuals in our study group undergoing VIT against the wasp (2.7\%) and in 6 patients allergic to bee venom (17.65\%). Only in one case, the administration of adrenaline was needed. Thus, the incidence of systemic adverse reactions during VIT in our cohort may be estimated to reach 1 event per 706 injections (1.4\% of injections), and potentially lifethreatening reactions (defined as a need of adrenaline treatment) as 1 per 7060 injections (0.14\% of injections). These findings are in line with a study by Ruëff et al. [18] where systemic adverse reactions were observed in $6-7 \%$ of patients undergoing VIT, but only in a few cases adrenaline was necessary $[19,20]$. No fatalities due to VIT have been described in the literature to date [16]. In our study majority of systemic reactions were observed in the induction phase (1.9\% of injections), not in the maintenance phase $(0.19 \%$ of injections). This confirms findings of Mosbech et al. [13] who in a cohort of 840 patients undergoing VIT reported majority of systemic side effects (1.9\%) during dose increase, and not in the maintenance phase ( $0.5 \%$ of injections). We have seen most of the systemic reactions (68.2\%) within $30 \mathrm{~min}$ after the injection. All late systemic reactions (31.8\% of events) were restricted to skin changes (urticaria, flush, reddening of the skin).

More than $20 \%$ of our study cohort patients were on ACEi or $\beta$-adrenergic antagonists. Interestingly, treatment with $\beta$-adrenergic antagonists or ACE inhibitors did not increase the risk of any kind of adverse events during VIT, systemic adverse events during VIT and was not associated with more severe symptoms during field stings. This is in contrast to the findings of Ruëff et al. [9] who observed a cohort of 1532 patients and reported that ACE inhibitor medications were the strongest factors associated with more severe symptoms during sting challenge, followed by honeybee venom allergy and systemic allergic reaction during VIT, whereas double VIT and longer duration of therapy reduced the failure rate. It needs to be noted that the number of patients on anti-hypertensive medications in our study was probably too small to make any meaningful observations, as that was an observational, real-life analysis, not a hypothesis-driven and not powered based on a pre-defined hypothesis.

There are several limitations inherited in the design of our study. The study group consisted of only 180 patients however, most of the studies performed in one center analyze cohorts of around 100 patients [11, 12]. What is more important only 34 patients were allergic to bee venom, which may bias results of observations in this sub-group and may result in underestimation of statistical significance of our findings. On the other hand, all real life and epidemiological studies confirm that wasp venom allergy is more frequent, thus we believe that our results mimic the situation found in the every-day clinical practice. All patients undergoing venom immunotherapy at our center received oral antihistamines as a pretreatment before the ultra-rush induction phase and during the maintenance treatment. This is a well-accepted clinical practice, however it needs to be noted that this may result in underestimation of the number and/or severity of adverse events during VIT in our cohort. Reimers et al. [11] in a cohort of 57 patients on VIT due to honeybee venom allergy found that fexofenadine (in a dose of $180 \mathrm{mg}$ ) significantly reduced local allergic reactions and generalized symptoms of the urticaria and angioedema type, but did not mask the anaphylaxis. A higher frequency of sting allergy in females was found in our study. In most published studies there is a 60 : 40 male predominance. This raises questions of relative exposure to stings in males and females in this population, referral patterns, access to care, cultural biases or other, unknown reasons that might affect the gender ratio. Another limitation of our work is that we have not studied any other than slgE possible biomarkers (e.g. serum tryptase), and thus we were not able to include those parameters in our analysis. However, current guidelines do not recommend any other biomarkers in a longitudinal follow-up of venom allergic subjects.

\section{Conclusions}

In the population studied, we have found that wasp is more frequent than bee venom allergy. Venom immunotherapy is highly efficacious and safe, as most of the adverse events during the induction and maintenance phase are mild and local. Side effects of VIT are more common in subjects on bee VIT. Majority of systemic side effects are reported during the induction phase, within 30 min after the injection, and the risk of adverse effects is not increased in patients receiving ACEi or $\beta$-adrenergic antagonists. Beekeeping and female sex are associated with a higher risk of allergy to Hymenoptera venom.

\section{Conflict of interest}

The authors declare no conflict of interest.

\section{References}

1. Ruëff F, Wenderoth A, Przybilla B. Patients still reacting to a sting challenge while receiving conventional Hymenoptera venom immunotherapy are protected by increased venom doses. J Allergy Clin Immunol 2001; 108: 1027-32.

2. Reisman RE. Allergy to stinging insects. In: Patterson's Allergic Diseases. $7^{\text {th }}$ ed. Grammer LC, Greenberger PA (eds.). Lippincott, Williams \& Wilkins, Philadelphia 2009; 220-31.

3. Antonicelli L, Bilo MB, Bonifazi F. Epidemiology of Hymenoptera allergy. Curr Opin Allergy Clin Immunol 2002; 2: 341-6.

4. Bilo BM, Bonifazi F. Epidemiology of insect-venom anaphylaxis. Curr Opin Allergy Clin Immunol 2008; 8: 330-7.

5. Lockey RF, Turkeltaub PC, Olive ES, et al. The Hymenoptera venom study. Safety of venom immunotherapy. J Allergy Clin Immunol 1990; 86: 775-80. 
6. Bonifazi F, Jutel M, Bilo MB, et al. Prevention and treatment of hymenoptera venom allergy: guidelines for clinical practice. Allergy 2005; 60: 1459-70.

7. Bousquet J, Müller UR, Dreborg S, et al. Immunotherapy with Hymenoptera venoms. Allergy 1987; 42: 401-13.

8. Ruëff F, Przybilla B, Bilo MB, et al. Clinical effectiveness of venom immunotherapy for Hymenoptera venom allergy: a prospective observational multicenter study of the European Academy of Allergology and Clinical Immunology Interest group on insect venom hypersensitivity. PLoS One 2013; 8: e63233.

9. Ruëff F, Vos B, Oude Elberink J, et al. Predictors of clinical effectiveness of Hymenoptera venom immunotherapy. Clin Exp Allergy 2014; 44: 736-46.

10. Müller U, Helbling A, Berchtold E. Immunotherapy with honeybee venom and yellow jacket venom is different regarding efficacy and safety. J Allergy Clin Immunol 1992; 89: 529-35.

11. Reimers A, Hari Y, Muller U. Reduction of side-effects from ultrarush immunotherapy with honeybee venom by pretreatment with fexofenadine: a double-blind, placebo-controlled trial. Allergy 2000; 55: 484-8.

12. Laurent J, Smiejan JM, Bloch-Morot E, Herman D. Safety of Hymenoptera venom rush immunotherapy. Allergy 1997; 52: 94-6.

13. Mosbech H, Müller U. Side-effect of insect venom immunotherapy: results from an EAACI multicenter study. Allergy 2000; 55: 1005-10.

14. Brehler R, Wolf H, Kutting B, et al. Safety of a two-day ultrarush insect venom immunotherapy protocol in comparison with protocols of longer duration and involving a larger number of injections. J Allergy Clin Immunol 2000; 105: 1231-5.

15. Wenzel J, Meissner-Kraemer M, Bauer R, et al. Safety of rush insect venom immunotherapy. The results of a retrospective study in 178 patients. Allergy 2003; 58: 1176-9.

16. Boyle RJ, Elremeli M, Hockenhull J, et al. Venom immunotherapy for preventing allergic reactions to insect stings. Cochrane Database Syst Rev 2012; 10: CD008838.

17. Jennings A, Duggan E, Perry IJ, Hourihane JO. Epidemiology of allergic reactions to Hymenoptera stings in Irish school children. Pediatr Allergy Immunol 2010; 21: 1166-70.

18. Ruëff F, Przybilla B, Biló MB, et al. Predictors of side effects during the buildup phase of venom immunotherapy for $\mathrm{Hy}$ menoptera venom allergy: the importance of baseline serum tryptase. J Allergy Clin Immunol 2010; 126: 105-11.

19. Birnbaum J, Ramadour M, Magnan A, Vervloet D. Hymenoptera ultra-rush venom immunotherapy (210 $\mathrm{min}$ ): a safety study and risk factors. Clin Exp Allergy 2003; 33: 58-64.

20. Sturm G, Kränke B, Rudolph C, Aberer W. Rush Hymenoptera venom immunotherapy: a safe and practical protocol for high-risk patients. J Allergy Clin Immunol 2002; 110: 928-33. 